\title{
Imágenes de imperio, erudición e historia en la nomenclatura lunar del siglo XVII
}

\section{Nydia PINEDA DE Ávila Universidad Nacional Autónoma de México}

Este artículo discute los procesos y criterios mediante los cuales los autores de las nomenclaturas lunares del siglo XVII desarrollaron una variedad de estrategias lingüísticas y retóricas para organizar y representar sus estudios telescópicos de la superficie lunar como espacio cartográfico. A través de una comparación de los sistemas toponímicos propuestos por Pierre Gassendi, Michael van Langren, Johannes Hevelius y Giambattista Riccioli, planteo que las toponimias de la Luna exhiben estrategias de nombramiento asociativas, analógicas, metafóricas y emblemáticas que derivan de erudición académica, prácticas cartográficas y contextos sociales. Estas nomenclaturas sirvieron para proyectar inquietudes políticas, filosóficas y disciplinarias de sus autores.

PALABRAS CLAVE: selenografía, analogía Tierra-Luna, toponimia y nomenclatura, retórica visual, estrategias mnemotécnicas.

This paper discusses the processes and rationale through which authors of lunar nomenclatures in the seventeenth century developed a variety of linguistic and rhetorical strategies in order to organise and represent their telescopic studies of the lunar surface as a cartographic space. Through a comparison of the naming systems advanced by Pierre Gassendi, Micheal Van Langren, Johannes Hevelius and Giambattista Riccioli, I argue that lunar toponymies display associative, analogical, metaphorical and emblematic naming strategies that derive from scholarly erudition, cartographic practices and social contexts. These nomenclatures were used to project political, philosophical and disciplinary concerns of their authors.

KEYWORDS: selenography, analogy Earth-Moon, toponymy and nomenclature, visual rhetoric, mnemotechnical strategies.

CALIBAN: Has thou not dropped from heaven?

STEPHANO: Out o'th moon I do assure thee. I was the man i'th'moon, when time was.

CALIBAN: I have seen thee in her, and I do adore thee. My mistress showed me thee, and thy dog, and thy bush.

STEPHANO: Come, swear to that: kiss the Book. I will furnish it anon with new contents. Swear. 
TRINCULO: By this good light, this is a very shallow monster. I afeared of him? A very weak monster. Well drawn, monster, in good sooth.

Shakespeare, The Tempest, II, 2, 135.

En tiempos de Shakespeare, el hombre en la Luna, "man in the moon", patrono nocturno de los borrachos, era un nombre común de las tabernas en Londres y un ícono de una religión burlesca (Urban, 1976: 203-205); al mismo tiempo, ésta era una figura que una niña podía dibujar en el espacio nocturno para enseñarle las partes del cuerpo celeste a un salvaje. No obstante, la analogía entre la mancha en la Luna y un hombre, aunque lúdica y directamente referencial, carecía de especificidad: era un topónimo desperdiciado. El hombre en la luna no era un recurso lingüístico suficientemente preciso para establecer y comunicar observaciones astronómicas. Aunque hay evidencia de que la imagen fue usada por astrónomos antes y poco tiempo después del desarrollo del telescopio (Whitaker: 6; Gassendi, Epistolae: 77, Opera Omnia IV: 278), en la comunicación científica del siglo XVII esta analogía fue sustituida por sistemas de nomenclatura que ayudaron a inventar un nuevo espacio cartográfico. Los astrónomos, al reconocer las posibilidades del telescopio para la ciencia, conscientes de prácticas cartográficas antiguas y modernas, descubrieron que al nombrar partes de la superficie lunar podrían reorganizar espacios desconocidos y apropiárselos desde su tradición intelectual y su imaginario cultural, para así usar el satélite en sus agendas intelectuales y políticas.

Este estudio ofrece una perspectiva de los contextos y procesos que llevaron al establecimiento de las nomenclaturas lunares concurrentes en la segunda mitad siglo XVII, cuando el telescopio como instrumento de investigación astronómica penetró en las culturas eruditas y los circuitos de intercambio europeo a través del mundo (Van Helden, 1974: 38-58). La discusión de este tema no ha sido frecuente dentro de los estudios literarios. En cambio, las escasas investigaciones acerca de toponimias lunares han sido desarrolladas por astrónomos interesados en la historia de su disciplina, por historiadores de la cartografía o en el área de la historia y epistemología de la geografía. Para astrónomos como Ewen Whitaker, las nomenclaturas lunares del siglo XVII son sistemas arcaicos de la taxonomía contemporánea del satélite, que resultaron de prácticas de observación y registro limitadas por la tecnología óptica y por la idiosincrasia de los observadores (1999: 17-80). Para Peter van der Krogt, especialista en la historia de la publicación de mapas, el interés de este tema radica en documentar un capítulo de la historia de la toponimia identificando los criterios clasificatorios propuestos en diferentes sistemas contemporáneos (1853-1855). Por otra parte, para el geógrafo cultural Christian Jacob, la nomenclatura lunar del siglo XVII constituye un ejemplo de cómo las inscripciones físicas en los mapas reflejan estrategias de construcción y representación del saber en el discurso geográfico ("La Cartographie au télescope": 606-633). En diálogo con estos acercamientos, los estudios literarios pueden ofrecer un enfoque que destaque 
la construcción de un espacio retórico a través del encuentro de palabra e imagen. Bajo esta perspectiva, es relevante entender al cartógrafo lunar o selenógrafo, como un autor formado en una cultura que fomentaba el uso adecuado de recursos retóricos como la analogía, la metáfora y el emblema para imprimir imágenes evocativas en la mente de interlocutores y lectores. En los siguientes ejemplos ilustraremos cómo los selenógrafos del siglo XVII crearon, a través de una variedad de estrategias retóricas, alusiones a su mundo geopolítico e intelectual en la cartografía lunar.

\section{La caja de herramientas: estrategias textuales para nombrar el espacio lunar}

En 1644, Pierre Gassendi (1592-1655), astrónomo francés y profesor del Collège Royal, le escribió a Juan Caramuel Lobkovitz (1606-1682), filósofo natural español de la orden cisterciense que planeaba publicar un mapa de la luna, para compartirle una observación astronómica. En su carta explica que, habiendo dedicado más de diez años al proyecto de publicar su propio mapa, a su edad se sentía incapaz de proseguir en la supervisión de artistas-grabadores o en la invención de nombres para identificar las regiones sobre el disco lunar; sin embargo, convencido de la utilidad de la selenografía, le compartía a su seguidor el vocabulario que había diseñado para identificar las manchas de la luna para que él lo siguiera desarrollando:

Homme très célèbre, si d'aventure tu veux connaître la petite observation du quadrilatère des Pléiades occulté par la lune que je transmets à Wendelin, tu reconnaîtras là une utilité de la sélénographie. Pour la tâche que j'appelle Caspienne, tu verras que je peux parler de celle qui est solitaire sur le bord à l'ouest, et à laquelle correspond presque à l'opposite une autre tâche semblable (on peut l'appeler Anti-caspienne). Tu en verras une autre que j'appelle Homoncule (ou ailleurs Thériste), je l'appelle aussi Mer de l'Ouest; par ailleurs, je nomme Mer de l'Est la plus grande que se répand vers l'est, et Mer du Nord celle qui se répand ver le nord; et de temps en temps aussi j'appelle la plus grande tâche à l'imitation des auteurs antiques, Sanctuaire d'Hécate. Pour ne rien dire de la désignation des parties plus petites, comme celle des Salines, de la Vallée Ombreuse, de la Roche de Neige, de la Montagne de la Mer Amère, du Nombril, de la Lagune et de semblables, que tu sauras sans aucun doute désigner avec plus de bonheur (Gassendi, Lettres Latines, 2004: 352-353).

El acto de nombrar, mediante diferentes estrategias semánticas que reflejan experiencias, creencias, cultura e historia, transforma un dibujo o un levantamiento territorial en un mundo trazado y organizado. Este proceso, explica Christian Jacob, puede nutrirse de una combinación de recursos léxicos que consisten en operaciones lingüísticas simplemente denotativas u otras de gran complejidad simbólica (The Sovereign Map: 203-238). El pasaje epistolar de Gassendi despliega, en este sentido, un cúmulo de material semántico: Mar del Este, Mar del Occidente y Mar del Norte, por ejemplo, 
derivan de prácticas cartográficas que asignan nombres relacionados con coordenadas geográficas, es decir, con atributos de localización u orientación de un punto en el espacio. Esos topónimos pueden también ser comparados a nombres dados a tierras desconocidas, como Terra Australis, en la tradición geográfica. Otros apelativos usados por el astrónomo derivan de analogías de aspecto: la forma de la mácula Laguna sería comparable a la de una formación hidrológica de este tipo en la convención cartográfica contemporánea del autor. En el catálogo de topónimos de Gassendi también figuran citas de autores clásicos; los nombres Santuario de Hécate y mácula Caspia son extraídos el diálogo de Plutarco De Facie in orbis lunae apparet (2013: 81, 83). Este diálogo, en español conocido como Sobre la cara visible de la Luna, propone dichos topónimos en un debate acerca de la naturaleza de la Luna (Coones, 1983: 361-372). Considerando esta variedad discursiva, es notable que la estrategia de Gassendi no era fijar una cuadrícula de nombres sino proponer una variedad de referentes para orientar la mirada del astrónomo en el espacio. Al proponer hasta dos o tres formas de identificar un mismo punto, Gassendi sugiere que su lista no es una taxonomía sistematizada sino una caja de herramientas lingüísticas personales que compartía con sus interlocutores.

A pesar de la heterogeneidad en este discurso toponímico, un aspecto relevante y coherente en el sistema de Gassendi se halla en la denominación de elementos topográficos, como mares y valles, que aluden a la presencia de una "geografía" lunar. Vale la pena detenerse en este aspecto clasificatorio que surge de una analogía entre la Tierra y la Luna, y que permea desde las nomenclaturas lunares del siglo XVII hasta nuestros tiempos. Queda fuera de los alcances de este estudio detallar el debate en torno a la analogía Tierra-Luna en el siglo XVII, por lo que basta recordar algunos puntos clave: la comparación entre la Tierra y su satélite es transmitida, sobre todo, a través del diálogo de Plutarco, que es referido indirectamente por Gassendi a través de algunos de sus topónimos. En este autor, ampliamente leído en el Renacimiento y la primera modernidad a través de la traducción de su Moralia (Hale Shackford, 1929: 16-17), la investigación astronómica encontró un imaginario, un vocabulario y un útil marco conceptual.

He aquí una breve síntesis de cómo se presenta la idea de la analogía Tierra-Luna en De Facie in orbis lunae apparet: al inicio, se alude a la hipótesis de que el gran océano se refleja en el disco lunar. Enseguida, esta imagen del espejo es rebatida con un argumento que defendía la naturaleza idéntica de la Tierra y su satélite: la Luna, como la Tierra, sería sólida y oscura, y no formada por la materia incorruptible de los astros según la doctrina aristotélica. Dichas características permitían al satélite reflejar la luz del Sol, al igual que el globo terrestre. Los defensores de esta idea extrapolan las implicaciones de esta analogía a través de un razonamiento matemático y óptico: como la luz se refleja en las superficies sólidas bajo ciertos ángulos, las regiones más brillantes del astro serían tierras; y, por otra parte, tomando en cuenta que la luz se refracta en el agua, los contornos y las honduras negras de la cara lunar serían aguas (Plutarco, 2013: 24-28). Al final del texto, en un mito acerca del ascenso de las almas de los di- 
funtos narrado por Sulla, el globo lunar es comparado con un mundo semejante al Mediterráneo (83). En este texto los astrónomos encontraron tanto un discurso óptico como una serie de recursos lingüísticos y poéticos para su comunicación científica.

La recepción de las imágenes y conceptos leídos en la obra de Plutarco fueron de gran utilidad para la traducción de observaciones e hipótesis generadas con el auge del telescopio. Galileo, como es bien sabido, recurrió a la analogía Tierra-Luna para divulgar sus investigaciones lunar es en el Sidereus Nuncius, publicado en Venecia en $1610(7 \mathrm{v})$. Aunque dicho autor es quizás considerado como el abogado más importante de la similitud Tierra-Luna en el discurso astronómico de su tiempo, es ya sabido que éste ensayó un topos muy frecuente tanto en la filosofía natural como en el discurso pictórico de sus contemporáneos (Fabbri, 2013: 103-135; Ariew, 1984: 213226). En cambio, en la obra de Kepler, gran lector y traductor de Plutarco quien respondió críticamente al famoso panfleto de Galileo, se halla una propuesta más elaborada y precisa de la analogía Tierra-Luna y de una geografía lunar (Ad vitellionem paralipomena: 150; Kepler's Conversation with Galileo's Sidereal Messenger: 13, 24, 26; Narratio: [*6]; Aït-Touati: 45-77). Esta idea se desarrolla notablemente en su Sueño u obra póstuma de astronomía lunar, texto en el cual, mediante una concatenación de alegorías, se construye una imagen mental de la topografía lunar en el lector (80-96). Esta obra es la fuente más importante de la divulgación de la palabra "selenografía" en la República de las Letras.

En la obra de astrónomos como Kepler, Galileo y muchos de sus contemporáneos (Gassendi, Viri illustris: 302-303; Hevelius, 1647: 206-223) es posible vislumbrar que la analogía Tierra-Luna, además de ser un instrumento retórico anclado en la autoridad de los filósofos clásicos, fue una herramienta heurística fundamental en la observación astronómica pues el telescopio no bastaba para entender e interpretar la imagen vista a través de la lente. La construcción de la selenografía requería tanto de lentes bien talladas y debidamente colocadas, como de buenas técnicas de registro, una memoria aguda, excelente capacidad de síntesis, una mano artista y, además, marcos epistemológicos para guiar la interpretación de la imagen. La analogía geográfica y óptica permitía leer diferentes grados de luz y sombra con métodos semejantes a aquellos usados durante un estudio topográfico.

Es fundamental subrayar que la concepción de la Luna como un mundo semejante a la Tierra tocaba un punto muy polémico en la cosmología de su tiempo. Específicamente, ponía en jaque la teoría de la quintaesencia, que definía a la Tierra y al mundo sublunar como las regiones corruptibles del universo, formadas por la mezcla de los cuatro elementos: agua, tierra, fuego y viento; a partir de la esfera de la Luna, este astro y todos aquellos en las órbitas más allá de él estaban constituidos por una substancia incorruptible. Proclamar que la Luna era semejante a su planeta más cercano demolía este sistema aristotélico del universo defendido en la filosofía escolástica y enseñado en universidades y colegios alrededor de Europa y sus colonias en el Renacimiento y la época del Barroco (Pantin, 2013: 103-120). 


\section{$30 \square$ IMÁGENES DE IMPERIO, ERUDICIÓN E HISTORIA EN LA NOMENCLATURA LUNAR}

Los selenógrafos, conscientes del debate intelectual en el cual sus imágenes estaban insertas, tomaron diferentes actitudes ante este conflicto. La lectura de sus escritos refleja, en términos generales, cierta cautela en la reivindicación de la similitud TierraLuna como una creencia cosmológica. Esta analogía, tan importante en la organización del mapa lunar como un mundo semejante al orbe de los atlas terrestres, era por lo general referida como una convención retórica útil para la consolidación de la imagen cartográfica de la Luna más que como una creencia (Hevelius, 1647: 148; Riccioli, 1651: 187). En este sentido, en la tensión entre hipótesis y convención, la imagen de la Luna como un mundo con mares, océanos, penínsulas, valles y montañas penetró en la cultura erudita del siglo XVII. En particular, la aceptación y la apropiación de la convención retórica permitió a los selenógrafos desarrollar una toponimia lunar basada en prácticas cartográficas históricas y coloniales, que permitía expresar sus agendas políticas e intelectuales.

\section{La luna como paisaje geopolítico y de circulación de saberes}

Nombrar un espacio recién descubierto colma un espacio vacío en el mapa, permite a un territorio ser visto y existir en el imaginario cultural (De Certeau, 1986: 143-144). El nombramiento del espacio geográfico es una forma de apropiación simbólica que otorga a un territorio un discurso histórico y una memoria. El Plenilunii Lumina Austriaca Philippica o "Luminarias austriacas filipinas de la Luna llena" de Michael van Langren (1598-1675), publicado en Bruselas es 1645, fue el primer ejemplo impreso de una cartografía lunar con nomenclatura detallada y con pretensiones de completud, y su título asocia directamente a la luna con el reino de Felipe IV, rey de España. En este discurso visual se encuentra un proyecto astronómico y una metáfora espacial: el mapa es presentado como un instrumento para la invención de un nuevo método de determinación de la longitud en tierra, que el autor explicó en el panfleto La Verdadera Longitud por mar y Tierra, publicado en español en 1644. Con este método Van Langren pretendía ganar un premio de seis mil ducados además de dos mil ducados de pensión vitalicia (Vyver, 1977: 81-84). Además de ser un aspecto técnico de este proyecto, el mapa de la Luna es una representación de la extensión de un imperio, un artificio retórico, concebido para complacer a un soberano. Las "luminarias austriacas filipinas", las regiones más visibles sobre el disco lunar, llevan nombres de hombres y algunas mujeres ilustres de esta dinastía.

Leyendo el mapa de derecha a izquierda, la región conocida como "el hombre en la luna" en el folclor y la tradición astronómica europeos es ofrecida a los Países Bajos: lo que sería la cabeza del hombre es llamado Mare Eugenianum, en honor a Isabel Clara Eugenia, Archiduquesa de Austria y regente de los Países Bajos durante el régimen español (1566-1633). Hacia el sur, este mar se abre hacia Mare Belgicum, y al suroeste toma el nombre de Sinus Batavicus, Golfo de los países neerlandeses. A la izquierda de esas aguas se halla un pequeño círculo al extremo del disco, llamado 
Manuel de Moura y Cortereal, gobernador de los Países Bajos de 1644 a 1647, quien, como indica el cartucho debajo del mapa, apoyó la publicación de esta cartografía. El espacio más vasto del globo lleva la inscripción Oceanus Philippicus en honor a Felipe IV de España, y las áreas en torno a éste representan su genealogía. Esta distribución de nombres demuestra un conocimiento de prácticas cartográficas coloniales en las cuales un territorio podía recibir, con consentimiento monárquico, el nombre de un soberano. Christian Jacob ha asociado estas prácticas a una forma de apropiación cultural que no sólo implica un símbolo de poder sino también un sentimiento de nostalgia y un deseo de identificación cultural con la tierra recién descubierta (The Sovereign Map, 2006: 205).

La selenografía de Van Langren, dirigida a interlocutores de toda índole, permite una lectura plurivalente. Aunque en esta imagen del imperio español sobre la luna sea posible identificar la percepción geopolítica de un cosmógrafo que desea obtener reconocimiento de su rey, también es legible una representación del tejido social dentro del cual la selenografía, como práctica del saber, se inserta: las "luminarias" más pequeñas de la carta llevan nombres de reyes, príncipes palatinos y condes italianos, que, como indica el cartucho del mapa, aluden a las redes mediante las cuales los patrones de las ciencias y las artes y sus clientes eruditos interactúan bajo la cultura del mecenazgo, un sistema de intercambios y beneficios recíprocos. Por otra parte, la toponimia apunta a un discurso filosófico y simbolismo ético latente en la imagen cartográfica.

El Plenilunii Lumina Austriaca Philippica designa a más de trescientas "luminarias" en la luna y contiene un catálogo de nombres emblemáticos de la República de las Letras desde el punto de vista de este cosmógrafo flamenco bajo el régimen español a mediados del siglo XVII. En el mapa es posible identificar patrones de la sociabilidad del autor: en la distribución de los nombres se pueden percibir algunos agrupamientos por nacionalidad, afinidad intelectual o incluso por amistad. Así, cerca de Mare Belgicum hay puntos que llevan nombres de los amigos del cartógrafo: Aestuaria Puteani Bamelrodia, por ejemplo, otorga un lugar en la Luna a Erycius Puteanus. Este gran corresponsal europeo en la primera mitad del siglo XVII, sucesor de Justus Lispsius (1547-1606) en la cátedra de latín del Collegium Trilingue de Louvain, fue el principal consejero de la retórica visual de Van Langren (Papy: 317-338). A lo largo de los meses precedentes a la publicación de la selenografía, este humanista comentó cada aspecto de la nomenclatura con el autor. Incluso, eligió el lugar que deseaba ocupar sobre ese nuevo globo. El 5 de marzo de 1645, le escribió:

J'aurai ainsi mon nom tout aussi bien placé, près de Mare Belgicum, là où cette mer forme un petit cercle en ressaut, non loin de notre ami Vendelinus qui se trouvera entre nous deux. Que par conséquent notre savant et bon Père Lafailli conserve sa place, ainsi que Monsieur Goetschoven bien digne d'avoir son point lumineux. Comme cette affaire et votre honneur me sont à cœur, je vous conseille de ne pas oublier Daniel Heinsius. C'est un des hommes les plus distingués de notre temps et qui peut devenir lui-même une des Lumières de la Lune. Plus il sera près de moi, plus cela me sera agréable (Bosmans, 1903: 128). 
Los hombres enlistados por Puteanus, cuyos nombres fueron inscritos en la selenografía, representan los lazos de solidaridad latentes en la nomenclatura. Lafailli (Jean della Faille, 1597-1652), matemático flamenco jesuita y cosmógrafo real de Felipe IV, y Vendelini (Geoffroi Wendelin, 1580-1667), astrónomo flamenco, eran antiguos colaboradores de Van Langren y le ayudaron a obtener el privilegio para la publicación de su mapa lunar. Pero la nomenclatura del Plenilunii Lumina Austriaca Philippica no es exclusivamente una documentación o una representación de lealtades y pertenencias. Al contrario, Puteanus aconsejó a Van Langren que trabajara por un sistema que fuera respetado por todos: "Il faut faire en sorte que nos ennemis ne puissent pas avoir prétexte pour faire une nouvelle carte de la Lune, après leurs vues" (130). Así, las inscripciones de esta cartografía incluyen tanto nombres de personajes que el autor consideraba amenazantes o en competencia con su proyecto, como Juan Caramuel Lobkowitz, quien, según Puteanus, estaba en Fráncfort a punto de publicar su propia selenografía mientras que Van Langren se apresuraba por hacer visible su empresa en Bruselas: "Voilà donc Caramuel à Frankfurt pour y faire graver sa carte de la Lune" (131).

La nomenclatura podría verse como un paisaje intelectual heterogéneo relacionado con experiencias, ambiciones y anhelos de un autor y sus colaboradores. Es posible ubicar comunidades eruditas formadas por maestros y alumnos, sobre todo en el caso de los matemáticos jesuitas cercanos al mundo de Van Langren: a un costado del golfo de la Geografía, Sinus Geograficus, al oeste de Mare Austriacum, el promontorio Clau se refiere al famoso director de la Academia de Matemáticas del Collegio Romano y profesor de los matemáticos jesuitas más distinguidos de su tiempo, Christophe Clavius (1538-1612). Por otra parte, al extremo opuesto de la misma bahía, Grégoire de St. Vincent, impulsor de la enseñanza de las matemáticas en los Países Bajos, tiene otro promontorio (P.S. Vincetti). Entre ambos se encuentra un punto que lleva el nombre Tacquetti, refiriéndose a André Taquet (1612-1660), estudiante de St. Vincent, profesor de matemáticas en Lovaina en 1645, año de la publicación de este mapa. Esta nomenclatura toma en cuenta a un buen número de estudiantes de St. Vincent, así como a otros jesuitas que circulaban entre los Países Bajos y el Colegio Imperial de Madrid, tales como como Joannes Ciermans, Thédore Moretus, Philippe Nuyts, Paul Guldin e Ignace Derkenni. Es relevante resaltar la representación de esta comunidad epistémica esbozada por Omer van Vyver (L'École de mathématiques des Jesuites: 266-277), ya que las inscripciones reflejan relaciones sociales e intelectuales del autor. Sin embargo, este discurso toponímico no es tan claramente un elogio al colegio jesuita. Los representantes de este grupo no son distinguidos como núcleos dominantes en el campo intelectual: profesores muy eminentes y censores de los colegios jesuitas como Christoph Grienberger y Guiseppe Biancani, célebres por sus escritos cosmológicos o por su rol en la edición de éstos (Feingold, Jesuits: 1-46; Gorman: 3-31), fueron colocados sin una lógica aparente, lejos de sus alumnos. Se puede decir que los matemáticos jesuitas no se encuentran representados en zonas cartográficas particulares, sino que comparten el territorio lunar con filósofos de diferentes tradiciones intelectuales. 
En esta nomenclatura también hay huellas de contactos entre redes de conocimiento, entre las cuales Van Langren no tenía relaciones directas o de fácil acceso: el nombre de Peter Cruger (Crugeri), profesor del astrónomo y selenógrafo más destacado del siglo XVII, Johannes Hevelius, fue sugerido por Puteanus puesto que aquel erudito le había enviado su obra impresa (Bosmans: 124). Por su parte, Hevelius, quien no tenía correspondencia con Van Langren pero en este tiempo ya distribuía pruebas de su propia selenografía (Pineda: 67-71), fue colocado cerca de Mare Borbonicum. La proximidad entre el nombre de Hevelius y ese topónimo que alude a la monarquía francesa podría asociarse también a una representación de circuitos de saberes conocidos por el cartógrafo: Van Langren sabía a través de corresponsales comunes como Pierre Gassendi, que Francia, y sobre todo París, era un punto importante para la recepción y la distribución de los trabajos de Hevelius a través de los círculos eruditos de éste y de Marin Mersenne. Pareciera que la inclusión de estos últimos en la nomenclatura representa las aspiraciones del cartógrafo de agradar y estrechar relaciones con éstos y otros actores semejantes de la República de las Letras.

En esta cartografía lunar se construye, a través de la nomenclatura, un paisaje intelectual difuso y, al mismo tiempo, cargado de tensiones y negociaciones. Es importante destacar que, aunque el agrupamiento por nacionalidades haya sido un criterio discutido entre Puteanus y Van Langren en el proceso del establecimiento de la toponimia, la distribución de nombres no siempre corresponde a nociones de pertenencia intelectual por país, religión o institución. Por otra parte, aunque la espacialidad, es decir, aspectos como la verticalidad, la horizontalidad, la proximidad y la distancia, jueguen un papel evidente en la construcción simbólica de la retórica visual, la ambigüedad cobra una función importante. Como se mencionó antes, el significado de la ubicación de puntos individuales en el espacio y las relaciones entre unos y otros no es claramente discernible. Posiblemente, los autores de la toponimia temían que una legibilidad demasiado clara desencadenara conflictos. Y, por otra parte, esta ambigüedad podía también servir para proteger una nomenclatura que evocaba personajes vivos y en una gran movilidad geográfica, intelectual e, incluso, religiosa. Es notable, además, que una segunda edición de esta carta fue publicada en 1670, con algunos nombres agregados a la nomenclatura; así, quizás éste fue un sistema taxonómico concebido como un discurso abierto a la actualización. ${ }^{1}$

Es importante subrayar otro nivel de lectura en este sistema: aquel que revela una mirada y una actitud hacia la historia. En la distribución de nombres sobre la superficie lunar se encuentra también una biblioteca; es decir, un acervo de nombres que representan ideas y obras de astrónomos antiguos. Así, siguiendo el consejo de Puteanus, al norte del globo lunar, en el Mare Astronomorum, Van Langren localizó a filósofos antiguos, tales como Xenofón, quien defendía la analogía Tierra-Luna (Bosmans: 130). Los nombres de los antiguos no sólo representan teorías a las cuales el autor quizás

\footnotetext{
${ }^{1}$ Quisiera agradecer a Martjin Stroms de la Biblioteca de la Universidad de Leiden por haberme informado de la existencia de esta edición, que se encuentra en una colección privada en Estados Unidos.
} 
adhería, sino a prácticas de lectura y de edición: a un costado de Aristarco, defensor de un sistema heliocéntrico, se puede leer el nombre de Pappus Alexandrinus (400 d. C.), el primer editor de su obra, así como el nombre del editor crítico entonces más reciente del filósofo griego, el matemático francés Gilles Personne de Roberval (1602-1675). El matemático Jean della Faille, en meses anteriores a la publicación del mapa, había dado noticia a Van Langren de las dificultades de este último para publicar otra edición crítica, la de la obra de Euclides, y el nombre de éste también se lee sobre el terreno lunar (Vyver, Lettres: 179). Así, discusiones y prácticas contemporáneas sobre textos, traducciones y ediciones se reflejan en la nomenclatura.

Por último, uno de los aspectos que amerita un comentario es la estrategia para la denominación de las tierras, las únicas zonas del satélite que no llevan nombres de personas. Según la correspondencia entre Van Langren y Puteanus, este aspecto del sistema toponímico fue de los últimos en acordarse. Por extensión del sistema anterior, Van Langren quería asignar nombres de monarquías a estas regiones. En cambio, Puteanus, para dar mayor variedad discursiva al mapa, sugiere la atribución de virtudes a estos espacios. Primero, sugiere Terra Pacis aludiendo a las negociaciones en curso para la conclusión de la Guerra de los Treinta Años (Bosmans: 129). Después, propone Terra Scientiae, Terra Laboris, Terra Virtutis y Terra Honoris. Sus consejos fueron tomados en cuenta y así se reflejan en la hoja impresa. A la lista fueron agregados Terra Dignitatis, Terra Iustitiae, Terra Sapientiae y Terra Temperantiae (Bosmans: 129-130). Estos nombres son emblemáticos de un discurso filosófico en el cual fue concebida esta cartografía.

De las virtudes representadas, el honor, el trabajo, la sabiduría y la virtud, sobre todo, pueden ser asociadas con la cultura erudita neoestoica que penetró en los Países Bajos a finales del siglo XVI y a lo largo del XVII, gracias a los trabajos de Justus Lipsius. Estos ideales, recomendados por Lipsius como principios de autorregulación y de gobierno de naciones, tuvieron una influencia importante en la iconografía de la cartografía contemporánea y pueden ser apreciados en la obra de Abraham Ortelius, amigo de Lipsius, en la de su colaborador Franz Hogennerg, y en la obra y correspondencia de artistas contemporáneos a Van Langren, como Peter Rubens (Morford: 7; Ettinghausen: 94-100; Nutti: 38-55; Mangani: 59-83). El elogio de la moral neoestoica en este mapa lunar puede explicarse como un reflejo de los ideales de la cartografía que circulaban en los Países Bajos y por relaciones estrechas entre maestros y alumnos: el humanista Lispsius fue maestro del ya mencionado Erycius Puteanus, consejero de Van Langren e inventor de este rubro de la nomenclatura, y, por otra parte, Van Langen provenía de dos generaciones de cartógrafos neerlandeses. Las tierras lunares de Van Langren, a través de sus topónimos, dialogan con este contexto intelectual y a través de ellas el mapa Plenilunii Lumina Austriaca Philippica alcanza un nivel de significación ética: como emblema de la aspiración filosófica a la calma en tiempos de conflicto; este mapa exhorta a los príncipes y hombres del saber a que construyan la paz a través de la justicia, el trabajo y el honor. La Luna, en esta cartografía, es la idealización de un lugar en el cual la humanidad puede ser redimida a través de la contemplación y el estudio. 


\section{Un mapa histórico proyectado en la Luna}

Dos años posteriores a la publicación del Plenilunii Lumina Austriaca Philippica, Johannes Hevelius dio a conocer una nomenclatura basada sobre principios muy diferentes a aquellos que guiaron el sistema de Van Langren. En su obra Selenografía, el tratado sobre cartografía lunar más leído en el siglo XVII, publicado en Danzig en 1647, la luna es representada como un espejo de la geografía antigua. Esta obra de más de quinientas páginas, con cuarenta fases del satélite, cuatro mapas de la luna llena y dos templetes de sus rasgos principales, incluye un mapa, "Tabula Selenográfica", que presenta la topografía del satélite en el estilo de la cartografía regional de su época (226-227).

Hevelius comentó el proceso de establecimiento de su nomenclatura: afirmó que una clasificación universal era indispensable para establecer la observación colectiva necesaria y urgente para el progreso de la astronomía y la geografía. Sin una nomenclatura común, la organización y el cotejo de datos tomarían más tiempo que las observaciones mismas. El autor asociaba el proyecto de clasificar las regiones del disco lunar con la historia bíblica según la cual Adán nombró el mundo para hacerlo comprensible. El astrónomo recalcaba que, así como las descripciones geográficas necesitaban nombres de regiones, ciudades, pueblos, mares, ríos, montañas y valles, la selenografía necesitaba de una toponimia (223).

Hevelius, quien a lo largo de su vida se esforzó ante todo por completar la cartografía celeste del hemisferio norte, identificó su empresa con las prácticas de astronomía antigua que daban a las estrellas nombres de personajes excepcionales, como Hércules, Casiopea, Andrómeda, y Perseo. Siguiendo este modelo, contempló primero que las máculas de la luna llevaran nombres de observadores que él consideraba los más destacados de su tiempo: Oceanum Coperniceum, Oceanum Tychonicum, Mare Keplerianum, Lacum Galilaei, Palludem Maestlini, Insulam Scheinerianam, Peninsulam Gassendi, Montem Mersenni, Vallem Bullialdi, Sinum Wendelini, Promontorium Crugerianum, Fretum Eichstadianum, Dessertum Linnemanni (224). Estos topónimos, que fueron descartados aunque referidos por el autor en su texto como memoria de su proceso, reflejan la conciencia de que la toponimia lunar podría representar afinidades intelectuales, del mismo modo que en el sistema de Van Langren. Sin embargo, habría que constatar una diferencia entre el cartógrafo flamenco y Hevelius: mientras que para el primero la nomenclatura basada en nombres de dignatarios y filósofos reproducía prácticas de la cartografía colonial y merecía ser replicado, para Hevelius este patrón derivaba de la tradición clásica y estaba destinada al fracaso.

Consciente del valor simbólico de la asignación de nombres al espacio lunar y deseoso de evitar conflictos, celos o enojos, Hevelius decide retomar una imagen antigua, la de la Luna como Antictona o contra-tierra, y establece una comparación entre el mundo antiguo y la Luna. En vez de recurrir a prácticas coloniales, Hevelius ubica su proyecto en la historia de la geografía. El autor subraya que ha encontrado en la Luna formas semejantes a aquéllas representadas en la cartografía terrestre entre 
los grados décimo a decimonoveno de longitud y vigésimoquinto a sexagésimo de latitud, según la geografía de Ptolomeo (225-226), es decir, en el mundo antiguo en torno al Mediterráneo.

Como ha sugerido Christian Jacob, el uso de topónimos con un peso histórico ofrece al cartógrafo una estabilidad proveniente del sentido de familiaridad, homogeneidad y coherencia construido por la tradición (The Sovereign Map, 2011: 205). Concretamente, para Hevelius la nomenclatura de la geografía antigua resulta mucho más estable puesto que los topónimos modernos cambiaban constantemente debido a las guerras y a la colonización de su tiempo. En consecuencia, el autor explica que el uso de nombres antiguos ayudaría a su lector a memorizar la organización del espacio lunar (226).Además, como se ha mencionado arriba, para reforzar el discurso retórico, el mapa que lleva inscrita esta nomenclatura reproduce las convenciones gráficas de los mapas de finales del siglo XVI e inicios del XVII.

Las analogías que se establecen entre la Luna y las regiones en torno al Mediterráneo no son, pese a lo declarado, claramente legibles y pareciera más bien que Hevelius juega a reorganizar el mundo antiguo para comunicar sus observaciones telescópicas. Podría decirse que el marco más general de la organización espacial sí refleja la cartografía terrestre con cierta inmediatez: por ejemplo, el punto al extremo oeste del disco, Palus Moeotis (Mar de Asov), y el polo sur, Arabia, eran regiones consideradas limítrofes al occidente y al oriente del mundo antiguo. Por otra parte, el norte de la Luna, Regio Hyperboria, lleva un significante genérico, un punto cardinal; aquí Hevelius sigue una estrategia frecuentemente empleada para nombrar tierras desconocidas y que incluso podía ser utilizado para nombrar el norte del globo terrestre en la cartografía contemporánea. El extremo este del satélite no es marcado por las columnas de Hércules o el mar Atlántico, como sería esperado, sino por Africa Pars, aludiendo a la continuación de las tierras de África del norte conformadas por Egipto y Libia. En este marco asociativo, la Luna era un reflejo desfigurado del mundo antiguo, pero los límites, al menos, resultaban familiares.

De lado izquierdo de la Luna, en aquella mancha llamada Oceanus Philippicus por Van Langren, Hevelius trazó el Mare Mediterraneum. Parece que en ambos casos resultaba importante dar a esta mancha prominente un nombre con asociaciones claras y de valor cultural fuerte. El mar Mediterráneo en la Luna tiene claras alusiones a la cartografía terrestre: se asemeja al mar Mediterráneo representado por cartógrafos como Ortelius o Mercator, aunque es orientado con el este hacia el norte. Al interior de esa figura se leen nombres de las islas Maiorca, Ophiusa, Minorca y Ebisus, que los lectores identificarían fácilmente con las islas de las costas de España y el sur de Francia. Prolongando las expectativas de interpretación de los aficionados a los atlas entonces en boga, Hevelius asocia puntos elevados del satélite con islas volcánicas, como Corsica, y la isla central, Sicilia, contiene un círculo grande con la inscripción Aetna Mons. Tomando en cuenta que la nomenclatura de Hevelius contiene muchos volcanes, parece haber un diálogo entre esta representación cartográfica de la Luna y debates contemporáneos acerca de la formación del satélite y la Tierra. Aunque no es posible 
afirmar que esta toponimia refleje la creencia de su autor en un origen volcánico de la Luna, al menos puede decirse que este sistema clasificatorio contiene jeux d'esprit bastante polémicos, sobre la naturaleza lunar.

Al leer este mapa se encuentran muchas analogías evidentes, como la forma de Italia dentro del Mediterráneo, sin embargo, hay también alusiones oscuras que habrían intrigado a sus lectores, sobre todo a aquéllos con lagunas en su conocimiento de la geografía antigua. El reto de la lectura cartográfica sería tanto más desafiante cuanto que las analogías cartográficas no eran el único criterio de Hevelius. Según el autor, al no hallar una comparación evidente o, al menos, adecuada, decidía otorgar apelativos relacionados con características físicas del punto observado (226). Esta metodología, tomada de prácticas cartográficas antiguas, dio la pauta para nombrar Mons Porphyrites a un punto violáceo que recordaba a Hevelius a una piedra preciosa antigua de ese color, descrita por Plinio y que tenía su homólogo en la cartografía egipcia.

Después de explicar su sistema, Hevelius presenta su nomenclatura en una tabla: los topónimos están ordenados alfabéticamente frente a sus paralelos en la cartografía moderna. Este suplemento al texto, que refleja el interés en la toponimia antigua aumentado a partir del redescubrimiento de Ptolomeo en el siglo XV, imita las tablas de concordancia que establecían equivalencias entre nomenclaturas geográficas antiguas y modernas publicadas a finales del siglo XVI (Toscanella; Sophianos; Pirckheimer; Ortelius). La fuente principal de la tabla de Hevelius fue el Thesaurus Geographicus de Abraham Ortelius. Este diccionario de nombres fue producto de una serie de colaboraciones y transformaciones editoriales (Depuydt, 1997: 37): la primera edición del Theatrum Orbis Terrarum (1570) incluía una lista de topónimos antiguous (Antiqua Regionum... nomina explicata) firmada por Arnoldus Mylius. En la edición del Theatrum de 1574, esta sección se amplió y renombró Synonymia locorum sive populorum... appellationes et nomina. En 1578, la tabla fue revisada y editada como Synomymia geographica y la edición de 1579 del Theatrum incluyó una nueva versión de esta tabla llamada Nomenclator Ptolemaicus; así, el Thesaurus Geográficus publicado en 1587, compilado a lo largo de este proceso, reeditado y expandido en 1596 por la industria plantiniana, cobró una gran presencia tanto en el mundo editorial como en la memoria de sus lectores.

Del Thesaurus Geographicus de Ortelius, Hevelius elige topónimos, sintetiza entradas, agrega definiciones propias y otras provenientes de fuentes especializadas en ciertas regiones tales como el renombrado Rerum Moscovitarum Commentarii de Sigismud von Herbertstein, publicado en Basilea en 1556. Esta alusión a las fuentes de la nomenclatura de Hevelius no sólo tiene interés documental; el uso del diccionario de Ortelius revela un aspecto importante del espíritu del proyecto toponímico del autor de la Selenographia: para Ortelius, la cartografía era un lugar de memoria, un ojo al mundo antiguo y sagrado, y su diccionario era una puerta de entrada a ese espacio intelectual y espiritual (Nutti: 44; Crosgrove: 856). La tabla selenográfica de Hevelius 
puede considerarse como un proyecto intelectual semejante. Los nombres de la geografía antigua son puntos de entrada a la meditación histórica y del lugar del ser humano en ella.

Entendiendo la lista de topónimos como clave de lectura para el soporte gráfico, la nomenclatura lunar cobra una dimensión adicional: la distribución de nombres no sólo sirve como instrumento de orientación para el observador en el registro de sus observaciones, sino también como un recordatorio de lugares y episodios emblemáticos de la antigüedad clásica y de la tradición bíblica, un lugar de memoria y contemplación de la historia de la Tierra. La presencia de una geografía sagrada, sobre todo en el hemisferio sur del globo, amerita un breve comentario pues la Luna de Hevelius puede ser leída como un espejo de la geografía bíblica, un tema exitoso en las culturas del libro impreso de la Reforma y la Contrarreforma.

La decisión de asociar todo el hemisferio sur de la Luna con territorio de las andanzas de los profetas no puede pasar inadvertida: las regiones del disco que llevan los nombres de Arabia, Aegyptus y Asia Minor son topónimos que aluden a narrativas de revelación, milagros, exilios y peregrinajes. La prominencia más grande del sur del hemisferio lunar, un trazo circular rodeado de líneas de montañas, Mons Sinai (ahora conocido como el cráter Tycho), relaciona este punto orográfico del satélite con el lugar simbólico de la Alianza bíblica de Moisés. Los topónimos en torno a este punto también son nombrados en episodios bíblicos del Antiguo Testamento. Por otra parte, los nombres del hemisferio norte pueden ser asociados al Nuevo Testamento, sobre todo a aquellos libros que relatan las misiones apostólicas. La proyección de la geografía sagrada sobre la Luna mediante esta distribución de topónimos no puede ser disociada de los esfuerzos por cristianizar el cielo en el siglo XVII, cuando un número de cartógrafos celestes transfirieron narrativas bíblicas a las estrellas (Warner: 224232; Kanas: 156-157; Brown: 31-33, 35). Por ejemplo, en el atlas Coelum Stellatum Christianum, de Julius Schiller, abogado católico en Augsburgo, publicado en 1627, se sustituyen las constelaciones ptolemaicas tradicionales por personajes y episodios bíblicos. Los doce apóstoles representan los signos del zodiaco en el hemisferio norte, y personajes e imágenes del Antiguo Testamento, como la emblemática Arca de Noé, son colocados al sur. Esta nomenclatura, establecida en colaboración con los jesuitas Johan Baptist Cysat, Paul Guldin, Jeremias Drexel y Mathew Rader, entre otros, fue un proyecto de la Contrarreforma. Otro ejemplo de este entusiasmo, aunque en un medio protestante, se halla en la figura de Wilhelm Schickard, luterano, matemático y profesor de lenguas antiguas, y autor del Astroposcopium pro facillima stellarum cognitione noviter excogitam, publicado en Tubingen en 1623. En esta obra Schickard agregó figuras bíblicas a las constelaciones ptolemaicas y su mapa es un sincretismo de tradiciones grecolatinas y cristianas. La reproducción de una geografía sagrada en la Selenografía de Hevelius puede ser entendido como un caso más de estos proyectos que buscaron renombrar los cielos y de crear nuevos instrumentos mnemotécnicos para la ciencia y la historia cultural. 


\section{Una nomenclatura como defensa de la astronomía en una historia institucional}

Las nomenclaturas de Van Langren y Hevelius representan, como se ha dicho, dos actitudes opuestas hacia el acto de nombrar el espacio celeste. En respuesta a dichos acercamientos contemporáneos, el filósofo natural Giambattista Riccioli presentó una toponimia alternativa en su obra enciclopédica Almagestum Novum, publicada en Bolonia en 1651. En el capítulo dedicado a la Luna (184-285), este jesuita incluye una nueva selenografía en dos hojas, la representación de cuatro fases, y una nueva nomenclatura en tablas (204-205). El espíritu de síntesis impregna esta empresa de representación lunar, al igual que integridad de su obra enciclopédica.

La selenografía de Riccioli constituye el proyecto de recolectar todas las informaciones disponibles sobre la materia lunar y de representarlas visual y textualmente. Las imágenes son precedidas por una scholia, un resumen de los estudios lunares antiguos y contemporáneos, así como por la explicación de los criterios tomados en la construcción de la imagen y el establecimiento de la nomenclatura. El proceso de la fabricación de la cartografía nueva se hace visible al lector en la presentación misma de ésta en dos hojas. La primera, sin texto, es ilustrativa de la metodología mediante la cual se construyó la imagen. El título explica: "Selenographia P. Francisci Mariae Grimaldi Soc. Iesu Optimo ex pluribus telescopio Lunae phasibus selecta, in qua Langreni, Hevelii, Eustachii, Sirsalis, etc. Selenographia partim firmavit, partim ita correxit, et auxit, ut vel minimce particulce ex aliquibus phasibus evidentiam sit assecutus" (204). ${ }^{2}$ Esta nueva selenografía, hecha con varios telescopios y una selección de fases, es una síntesis aumentada y corregida de ejemplos precedentes. Ahora bien, la segunda hoja contiene un mapa detallado que da prueba de resultados innovadores de estas investigaciones astronómicas: Figura pronomenclatura libratione lunari (204) es una cartografía con una toponimia expandida que muestra los puntos extremos del disco, registrados por primera vez tras un largo estudio de los movimientos libratorios, es decir, de las sutiles oscilaciones longitudinales y latitudinales del satélite. Estos nuevos puntos resultaban útiles para perfeccionar la determinación de la longitud en tierra. La nueva selenografía tuvo una amplia recepción entre sus contemporáneos y el sistema toponímico propuesto por Riccioli es la base de la nomenclatura lunar actual.

Bajo el título de Figura pronomenclatura libratione lunari, las leyendas indican una postura filosófica latente en el mapa: Nec homines incolunt y Nec anime lunam migrant. El autor de esta representación se aleja de forma manifiesta de las teorías que postulan la analogía Tierra-Luna y utiliza la comparación únicamente como convención

\footnotetext{
2 "Selenographia hecha por el padre de la Sociedad de Jesús, Franciso María Grimaldi, mediante un telescopio excelente, seleccionada de varias fases lunares, la cual en parte confirma las selenografías de Langren, Hevelius, Eustaccio Sirsali, etc., y en parte las corrige de tal manera que incluso las partículas mínimas de sus fases pueden ser mostradas". [La traducción es mía.]
} 
toponímica. Esta nomenclatura, había anticipado el jesuita, no seguiría el modelo de Hevelius puesto que lo encontraba cargado de analogías erróneas. Incluso insistía en que la analogía Tierra-Luna era una convención que podía desencadenar confusiones y reforzar creencias filosóficas inexactas. Su nomenclatura tomaría, en cambio, nombres de personas, como en el sistema de Van Langren. Sin embargo, su idea era crear un espacio discursivo que hablara directamente de la disciplina astronómica. Así, distribuyó en el mapa del disco lunar nombres de astrónomos y astrólogos antiguos y modernos sobre un fondo astrometeorológico. Esto daba a la analogía Tierra-Luna una connotación totalmente diferente a los ejemplos selenográficos anteriores.

Inmediatamente después de la presentación de las dos selenografías, Riccioli ofrece a sus lectores dos tablas comparativas de las nomenclaturas lunares contemporáneas: Nomenclatura lunarium partium y Nomenclatura Lunarium regionum (204). Estas listas no sólo constituyen un inventario de nombres sino una genealogía de las nomenclaturas lunares del siglo XVII. Al oeste del disco, en la región que Van Langren llamó Oceanus Philippicus y Hevelius Mare Mediterraneum, Riccioli colocó Oceanus Procelarum, mar de las tormentas, y alrededor de este espacio se lee península deliriorum (península de los delirios), península fulgrum (península de los rayos), mare humorum (mar de los humores), sinus epidemiarum (golfo de las enfermedades), mare imbrium (mar de las lluvias) e insula ventorum (isla de los vientos). Estos topónimos, alusivos a estados temperamentales y meteorológicos asociados con la agitación, la inestabilidad y el declive, cargan las latitudes occidentales de la Luna de un simbolismo negativo. Este tono del discurso continúa hacia el norte, cubierto por topónimos con un campo semántico asociado a la muerte y al frío. Esta connotación del terreno cartografiado encuentra su contraparte en el este de la Luna, donde las inscripciones toponímicas como mare tranquilitatis (mar de la tranquilidad), mare nectaris (mar de la dulzura), mare fecunditatis (mar de la fecundidad) y mare vigoris (mar del vigor) evocan atributos positivos. Pareciera entonces que el cuerpo lunar se restituye visualmente como un espacio que refleja los aspectos positivos y negativos de la condición humana. Los nombres que Riccioli asigna a las aguas representan teorías que establecen lazos estrechos entre los movimientos de los astros, la meteorología terrestre y los temperamentos humanos. Este aspecto de la nomenclatura puede ser asociado con las prácticas de taxonomía escolástica que clasifican el mundo bajo sistemas lógicos de causa-efecto (Crombie, 1994: 1245-1292).

Riccioli agregó un segundo nivel sobre este discurso visual: un catálogo de nombres que pertenecen a la historia de la astronomía. El jesuita explica que para organizar la distribución dividió el círculo en octantes. Leyendo el mapa en el sentido de las manecillas del reloj, el primero y el segundo contienen a los physicoastronomi más antiguos. El resto de los antiguos se encuentra distribuido en los octantes tercero y cuarto, y al inicio del quinto y el sexto. Los astrónomos medievales, la mayoría proveniente del mundo árabe, están incluidos en el quinto octante. Finalmente, en los octantes quinto, 
sexto, séptimo y octavo están dispersos los modernos. Así como Van Langren rindió homenaje a eruditos que debatían cuestiones cosmológicas, matemáticas y teológicas más allá de fronteras nacionales, políticas, religiosas e, incluso, temporales, Riccioli subraya que la organización de los nombres permitiría al lector establecer relaciones cronológicas y filosóficas (204). La nomenclatura de Riccioli, como la de Van Langren, es una visualización de una historia intelectual; ésta es una historia de la construcción de la astronomía como una disciplina con una tradición propia.

Es relevante destacar la función de esta selenografía y de su nomenclatura en la estructura del Almagestum Novum. En primer lugar, como encomio de la astronomía, la cartografía lunar refleja los contenidos del prefacio, la cronología y el catálogo de astrónomos, que preceden la exposición temática del primer tomo de la obra (i-xlvii). La defensa de la astronomía como una disciplina histórica y científica, así como la lista de astrónomos antiguos y modernos presentada por Riccioli en estos paratextos, es reformulada como una visualización muy sintética en la imagen cartográfica. El que esta historia disciplinaria sea contada en un mapa lunar es tanto más emblemático cuanto que la Luna, el cuerpo celeste más cercano a la tierra, fue el primer astro de estudio de la astronomía antigua y moderna, y el primer objeto contra el cual las tecnologías, las prácticas y las teorías se ponían a prueba.

La interpretación de esta visualización ha sido objeto de afirmaciones aceleradas y deterministas: ya que en el último octante, en el Mare Procelarum se leen los nombres de Hevelius, Galileo, Riccioli, Grimaldi, Kepler, Copérnico, Rheticus, Linneman, Cardanus y De Cusa, entre otros, y se sostiene que la localización de estos modernos denota la postura cosmológica del autor, ya sea la condena del modelo heliocentrista o su criptocopernicanismo, representado en una región cuyo nombre conlleva connotaciones conflictivas (Vertesi: 412; Dinis: 49-77). Habría, en cambio, que comprender la carga retórica de esta representación en el contexto de la educación jesuita. Como han mostrado los estudios de René Raphael, las prácticas de enseñanza jesuita favorecían el aprendizaje de diversas cosmologías, incluso de las más polémicas, con el fin de ofrecer a los estudiantes herramientas argumentativas para participar en debates en defensa de las doctrinas aristotélicas asociadas con su institución (419-440). Por otra parte, el Ratio Studiorum, la base regulatoria de la enseñanza jesuita, motivaba a los estudiantes a abrazar una cultura que valorizaba el emblema, el estudio de símbolos pitagóricos, jeroglíficos y otro tipo de enigmas (Findlen: 264). La selenografía de Riccioli sería, así, una invención retórica, un instrumento pedagógico mediante el cual se narra la historia de la astronomía de forma circular y sintética, por medio de un diagrama muy atractivo. En este sentido, esta selenografía, en el legado de la Apología pro Tychone contra Ursus de Kepler, puede ser ligada a los esfuerzos por legitimar la astronomía como una disciplina del saber (Jardine, 1984: 258-294).

El criterio de Riccioli, guiado por los consejos de Athanasius Kircher (1602-1680), era reunir en una sola obra fragmentos de autores de diferentes escuelas y actitudes filosóficas (Dinis, 2002: 205). Su objetivo no era manifestar sus creencias cosmoló- 
gicas sino exponer sintéticamente una historia de la astronomía como una disciplina del saber en la cual los jesuitas formaban parte activa. Así, en las regiones Terra Fertilitas, el sexto octante del disco lunar y el más densamente poblado, con cuarenta y nueve puntos nombrados, es posible leer una genealogía de matemáticos de la Compañía de Jesús. Entre los nombres gravados se hallan Christoph Calvius (15381612) y Christoph Griemberger (1561-1636), figuras clave en el desarrollo de la investigación matemática jesuita, y Guiseppe Biancani (1566-1624), profesor de Riccioli, todos ellos también presentes en la nomenclatura de Van Langren. De esta forma, en este mapa se puede ver también una historia institucional. Es por demás probable que Riccioli no destinara esta selenografía exclusivamente a una comunidad jesuita restringida, sino a un público diverso, pues sabía que su obra podría circular en las Indias a través astrónomos misionarios y por la intermediación de su asesor y protector Athanasius Kircher.

\section{Una metáfora de la acción humana más allá de la Tierra}

Este acercamiento a las nomenclaturas del siglo XVII muestra que, en el establecimiento de nombres de la topografía lunar, los astrónomos recurrieron a estrategias y vocabularios preexistentes en las tradiciones y las disciplinas del saber contemporáneas a su empresa. La variedad de acercamientos utilizados en la práctica toponímica de su tiempo se nutrió, de forma destacable, de patrones de lealtad e identidad, ideales filosóficos, diccionarios geográficos y teorías médicas, pero, sobre todo, se concibieron y promovieron taxonomías del espacio que reflejan compromisos políticos e intelectuales, creencias y aspiraciones.

Estas representaciones cartográficas, a través de la retórica de sus nomenclaturas, fueron usadas a finales del siglo XVII, más que en observaciones astronómicas regulares, en una comunicación científica que promovía a individuos e instituciones que participaban en el establecimiento y la legitimización de observatorios fundados con la misión de establecer meridianos nacionales. La asociación entre nomenclaturas lunares y proyectos de connotación nacionalista puede verse, por ejemplo, en las observaciones astronómicas publicadas en la revista londoniense The Philosophical Transactions durante 1675, año de la fundación del Royal Observatory of Greenwich. Éstas muestran que astrónomos franceses e ingleses expresaban su rivalidad y afirmaban sus identidades científicas locales mediante la adopción de nomenclaturas lunares distintivas; los franceses usaban el sistema de Riccioli en tanto que los ingleses se servían de la nomenclatura de Hevelius para publicar sus hallazgos (Oldenburg: 257269; 289-292; 371-372; 561-565).

Los casos estudiados en este ensayo revelan que las primeras taxonomías del espacio lunar eran el reflejo de las culturas en las cuales se desarrollaba la práctica astronómica. Estas formas de apropiarse del paisaje celeste, cargadas de negociaciones y anhelos, eran proyecciones, a menudo ambiguas, de marcos de referencia personales, 
institucionales y culturales forjados a través de estrategias textuales y cognitivas como la analogía, la alegoría y la metáfora. La Luna fue transformada, a través de sus nomenclaturas poéticas, en un símbolo de la extensión de la acción humana y de sus aspiraciones al espacio celeste. En este contexto, la selenografía no puede verse únicamente como objeto de especialización astronómica. Los mapas lunares eran, además de instrumentos para facilitar una observación colectiva, artificios textuales y visuales para acreditar a sus autores como individuos expertos o representantes de instituciones. En este sentido, la producción de la imagen cartográfica tiene que entenderse como un discurso retórico complejo que alude a las relaciones interdisciplinarias, a la colaboración de artistas, humanistas y astrónomos, al mercado del libro y a la cultura del mecenazgo que englobaba la creación intelectual, artística y tecnológica del siglo XVII.

\section{Obras citadas}

AïT-TOUATI, Frédérique. 2011. Contes de la Lune. Essai sur la fiction et la science modernes. París: Gallimard.

ARIEW, Roger. 1984. "Galileo's lunar observations in the Context of Medieval Lunar Theory”. Studies in History and Philosophy of Science, vol. 15, núm. 3. Pp. 213-226.

Bossmans, Henri. 1903. "La Carte Lunaire de Van Langren conservée aux Archives Générales du Royaume, à Bruxelles". Revue des questions scientifiques, vol. 4. Pp. 106-139.

Brown, Basil. 1932. Astronomical Atlases, Maps/Charts. An historical Guide. Londres: Search.

Certeau, Michel de. 1986. Heterologies: Discourse on the Other. Trad. Brian Massumi. Minneapolis: University of Minnesota Press.

CoOnes, Paul. 1983. "The Geographical Significance of Plutarch's Dialogue, Concerning the Face which Appears in the Orb of the Moon". Transactions of the Institute of British Geographers, vol. 8, núm. 3. Pp. 361-372.

Cosgrove, Denis. 2003. "Globalism and Tolerance in Early Modern Geography". Annals of the Association of American Geographers, vol. 93. Pp. 852-870.

CrombIE, Alistair. 1994. Styles of scientific thinking in the European tradition: the history of argument and explanation especially in the mathematical and biomedical sciences and arts, vol. 2. Londres: Duckworth.

DEPUYDT, Joost. 1997. “'Vale, verum antiquae historiae lumen': Antiquarianism in the correspondence between Justus Lipsius and Abraham Ortelius". Iustus Lipsius Europae Lumen et Columen. Proceedings of the International Colloquium 17-19 September 1997. Ed. G. TOURNOY, J. de LANDShEER y J. PAPY. Louvain: Presses Universitaires de l’Université de Louvain. Pp. 34-46. 
DinIS, Alfred. 2003. "Giovanni Battista Riccioli and the Science of his Time". Jesuit Science and the Republic of Letters. Ed. M. FEInGOLD. Cambridge: The Massachusetts Institute of Technology Press. Pp. 195-224.

2002. "Was Riccioli a secret Copernican?" Giambattista Riccioli e il merito scientifico dei Gesuiti nell'età Barocca. Ed. M. T. BorGATO. Florencia: Leo S. Olschki. Pp. 49-77.

Ettinghausen, Henry. 1971. "Neostoicism in Pictures: Lispsius and the Engraved Title-Page and Portrait in Quevedo's 'Epicteto y Phocilides"'. The Modern Language Review, vol. 66, núm. 1. Pp. 94-100.

FABBRI, Natacha. 2013. "The Moon as Another Earth: What Galileo owes to Plutarch". Galilaeana, vol. 9. Pp. 103-135.

FEINGOLD, Mordechai. 2003. "Jesuits: Savants". Jesuit Science and the Republic of Letters. Ed. M. FeInGOLD. Cambridge: The Massachusetts Institute of Technology Press. Pp. 1-46.

FInDLEN, Paula. 2003. "Scientific Spectacle in Baroque Rome: Athanasius Kircher and the Roman College Museum". Jesuit Science and the Republic of Letters. Ed. M. Feingold. Cambridge: Massachusetts Institute of Technology Press. Pp. 225-284.

Galilei, Galileo. 1992. Le messager céleste. Trad. Isabelle PAnTin. París: Belles Lettres.

1610. Sidereus Nuncius. Venetiis: Apud Thomam Baglionum.

Gassendi, Pierre. 2004. Pierre Gassendi (1592-1655), Lettres Latines I. Trad. Sylvie TAussig. Turnhout: Brepols.

. 1658. Epistolae [...] Tomus sextus. Lyon: Laurentii Anisson \& Ioannis Baptistae Devenet.

1658. Opera Omnia, vol. IV. Lyon: Laurentii Anisson \& Ioannis Baptistae Devenet.

1641. Viri illustris Nicolai Fabricii de Peiresc [...] Vita [...]. París: Jean Jacques Bouchard.

Gorman, Michael John. 2003. "Mathematics and Modesty in the Society of Jesus: The Problems of Christoph Grienberger". The New Science and Jesuit Science: Seventeenth Century Perspectives. Ed. Mordechai FEINFOLD. Dordecht / Boston / London: Kluwer Academic Publishers. Pp. 1-120.

Hale ShacKFord, Martha. 1929. Plutarch in Renaissance England with Special Reference to Shakespeare. [n. p]: Wesley College.

HeLdEN, Albert Van. 1974. "The Telescope in the Seventeenth Century". Isis, vol. 65, núm. 1. Pp. 38-58.

Herbertstein, Sigismud von. 1556. Rerum Moscovitarum Commentarii. Basilea: Johann Oporinus.

JACOB, Christian. 2011. "La cartographie au télescope: la lune, de Galilée à Riccioli”. Les lieux de savoir 2, Les mains de l'intellect. Ed. Christian JACOB. París: Albin Michel. 
2006. The Sovereign Map: Theoretical approaches in cartography throughout history. Trad. Tom ConLEY. Chicago: University of Chicago Press.

JARDINE, Nick. 1984. The birth of history and philosophy of science. Kepler's A defence of Tycho against Ursus with essays on its provenance and significance. Cambridge: Cambridge University Press.

Johannes Hevelius. 1647. Selenographia: sive, Lunce Descriptio. Gedani edita: Autoris sumtibus. Typis Hünefeldiani.

KanAS, Nick. 2007. Star Maps. History, Artistry and Cartography. Berlín: Springer / Chichister / Praxis.

KEPLER, Johannes. 1965. Kepler's Conversation with Galileo's Sidereal Messenger. Ed. y trad. Edward Rosen. Nueva York: Johnson Reprint Corporation.

Kepler's Somnium: the dream, or posthumous work on lunar astronomy. Ed. y trad. Edward Rosen. Madison / Londres: University of Wisconsin Press. 1634. Somnium seu opus posthumum de astronomia lunari. Fráncorft: Sumptibus haeredum authoris.

1611. Narratio de observatis a se quatuor Jovis Satellitibus erronibus. Fráncfort: Sumtibus D. Zachariae Palthenii.

1604. Ad vitellionem paralipomena, quibus astronomiae pars optica traditur. Fráncfort: Apud Claudium Marnium \& Haereded Ioannis Aubrii.

KRÖGT, Peter van der y Fermjan Ormeling. "Michiel Florent Van Langren and Lunar Naming". Actes del XXIV Congrès Internacional d'ICOS sobre Ciències Onomàstiques, Annex. Secció 8. Web. 2 de enero de 2018. <http://www. gencat.cat/llengua/BTPL/ICOS2011/190.pdf>.

LANGREN, Michael van. 1964. La verdadera longitud por mar y tierra. Amberes / Bruselas: [n. p.].

1645. Plenilunii Lumina Austriaca Philippica. Bruselas.

MANGANI, Giorgio. 1998. "Abraham Ortelius and the Hermetic Meaning of the Cordiform Projection”. Imago Mundi, vol. 50. Pp. 59-83.

Morford, Mark. 1991. Stoics and Neostoics. Rubens and the Circle of Lipsius. Princeton: Princeton University Press.

NuTI, Lucia. 2003. "The World Map as an Emblem: Abraham Ortelius and the Stoic Contemplation". Imago Mundi, vol. 55. Pp. 38-55.

OLDENBURG, Henry. 1676. "An extract of a Latin letter of Signor Cassini, containing both his considerations upon Mr. Flamsteed's account of the lunar eclipse of Decem. 21. 1675, and his own observations of the same eclipse". Philosophical Transactions, núm. 123, vol. 11. Pp. 561-565.

1675. "A more particular account of the last eclipse of the moon, as it was observed by the Parisian astronomers, and promised by us in our former numb. 111. English't out of the French Journal des Sçavants". Philosophical Transactions, núm. 112, vol. 10. Pp. 257-269.

1675. "Lunæ totaliter deficientis observationes, Londini in arce Londlnensi habitæ, idque in ædibus \& cum instrumentis Jonæ More Equ. Aurati, à 
Johanne Flamsteadio, astr. regio, nocte seqente d. Junii 26. 1675. st. vet”. Philosophical Transactions, núm. 116, vol. 10. Pp. 371-372. 1675. "Observatio Eclipseos Lunæ totalis cum occultationibus quarundam Fixarum, habita à Joh. Hevelio Anno 1675. die + , 11 Januarii St. n. Vesp”. Philosophical Transactions, núm. 113, vol. 10. Pp. 289-292.

PANTIN, Isabelle. 2013. "Le Débat sur la substance lunaire après le Sidereus Nuncius: Stratégie et Visée de la Résistance Péripatéticienne”. La lune aux 17 e et 18 e Siècles. Ed. Chantal Grell. Turnhout: Brépols.

PAPY, Jan. 2000. 'In Praise of the Omnipresent Egg: Erycius Puteanus' Ovi Encomium (1615)". Humanisitica Lovaniensia Journal of Neolatin Studies, vol. 49. Pp. 317-338.

PINEDA DE ÁvILA, Nydia. 2017. Selenographies in the seventeenth century: making, publishing and copying maps of the moon. Queen Mary University of London. [Tesis doctoral en literatura inglesa, dirigida por Markman Ellis].

PIRCKHEIMER, Willibald. 1585. Descriptio Germaniae utriusque tam superioris quam inferioris, auctoribus Bilibaldo Pirckheimero et coeteris. Antverpiae: ex officina C. Plantini.

PluTARQue. 2013. Le visage qui apparaît dans le disque de la lune. De facie quae in orbe lunae apparet. Intr. y trad. Jacques Boulogne. Dir. Alain LEROULD. Lille: Presses Universitaires du Septentrion.

Puteanus, Erycius. 1957. Honderd veertien Nederlandse brieven van Erycius Puteanus aan de astronoom Michael Florent van Langeren. Met een inleiding uitgegeven door J. J. Moreau. Ámsterdam: Antwerpen / Wereldbiblioteheek.

RAPHAEL, René. 2015. "Copernicanism in the Classroom: Jesuit Natural Philosophy and Mathematics after 1633". Journal for the History of Astronomy, vol. 46, núm. 4. Pp. 419-440.

Riccioli, Giambattista. 1615. Almagestum Novum. Bononiæ: Ex typographia hæredis Victorij Benatij.

ShaKeSPEARE, William. 2016. The Tempest. The Norton Shakespeare. 3a. ed. Nueva York: W. W. Norton \& Comp. Inc.

SIMAR, Theophile. 1909. Étude sur Erycius Puteanus, 1574-1646, considéré spécialement dans l'histoire de la philologie belge et dans son enseignement à l'Université de Louvain ... Ouvrage accompagné de pièces inédites et d'une planche hors-texte. Louvain: Presses Universitaires de Louvain.

SOPHIANOS, Nikolaos. 1570. Nomina antiqua et recentia urbium Graeciae descriptionis a N. Sophiano jam aeditae. Romae: typis A. Lafreri.

TOSCANELlA, Orazio. 1567. I Nomi antichi e moderni delle provincie, regioni, città, castella, monti, laghi, fiumi, mari, golfi, porti et isole dell'Europa, dell'Africa et dell'Asia, con le graduationi loro in lunghezza e larghezza et una breve descrittione delle suddette parti del mondo. Venecia: F. Franceschini.

Urban, Rayomd A. 1976. "Why Caliban Worships the Man in the Moon". Shakespeare Quarterly, vol. 27, núm. 2. Pp. 203-205. 
VERTESI, Janet. 2007. "Picturing the Moon: Hevelius's and Riccioli's Visual Debate". Journal for the History and Philosophy of Science, vol. 38. Pp. 401-421. Vyver, Omer van der. 1989. "L'École de mathématiques des Jésuites de la Province Flandro-Belge au XVII e Siècle". Archivum Historicum Societatis Iesu, vol. 49. Pp. 266-277.

1977. "Lettres de J.-Ch. della Faille, S. I., cosmographe du roi à Madrid, à M.-F. Van Langren, cosmographe du roi à Bruxelles, 1634-1645". Archivum Historicum Societatis Iesu, vol. 46. Pp. 73-183.

WARNER, Deborah J. 1979. The Sky Explored. Celestial Cartography 1500-1800. Nueva York / Ámsterdam: Theatrum Orbis Terrarum.

WHITAKER, Ewen. 1999. Mapping and Naming the Moon. Cambridge: Cambridge University Press. 